\title{
Statistical Image Reconstruction Methods for Simultaneous Emission/Transmission PET Scans
}

\author{
Hakan Erdoğan and Jeffrey A. Fessler \\ Dept. of EECS, University of Michigan
}

\begin{abstract}
Transmission scans are necessary for estimating the attenuation correction factors (ACFs) to yield quantitatively accurate $\mathrm{PE} T$ emission images. To reduce the total scan time, postinjection transmission scans have been proposed in which one can simultaneously acquire emission and transmission data using rod sources and sinogram windowing. However, since the post-injection transmission scans are corrupted by emission coincidences, accurate correction for attenuation becomes more challenging. Conventional methods (emission subtraction) for $A C F$ computation from post-injection scans are suboptimal and require relatively long scan times. We introduce statistical methods based on penalizedlikelihood objectives to compute ACFs and then use them to reconstruct lower noise PET emission images from simultaneous transmission/emission scans. Simulations show the efficacy of the proposed methods. These methods improve image quality and SNR of the estimates as compared to conventional methods.
\end{abstract}

\section{INTRODUCTION}

For accurate PET emission images, one must correct for effects of attenuation, or absorption of the gamma photons within the patient being imaged. The effects of attenuation on the final emission image quality are particularly severe in thorax imaging, since the larger dimension of the chest decrease the survival probability of photon pairs to as low as $2 \%$. There is potential new clinical applications for performing PET of the thorax, such as detection of breast cancer and lung tumors. Hence, the improvement of attenuation correction methods is getting more important.

The development of ring and rod sources for PET transmission scans made it possible to measure attenuation properties of a patient directly. Currently, attenuation correction factors (ACFs) are computed from a transmission scan that precedes the radiotracer injection, thereby increasing total scan time. Reducing the scan time is crucial to increase the patient throughput and to make attenuation corrected total body PET scans possible. For this purpose, post-injection measurements have been proposed that use rotating rod sources and sinogram windowing to simultaneously acquire transmission and emission sinograms. Simultaneous scans also eliminate the problem of misregistration between emission and transmission reconstructions.

Sinogram windowing is performed by taking a narrow window in the sinogram domain around the detector pair locations that are collinear with the rod source. This window captures the transmission coincidences, and is called the transmission window. There are also true emission events in the transmission window that must be corrected. The remaining sinogram bins, called the emission window, contain emission coincidences, scattered and random coincidences. The random events in the emission window are due both to emission and trans-

This work was supported in part by NIH grant CA-60711. mission events and are generally higher count as compared to a single emission scan [1].

Conventional methods of reconstruction used in simultaneous scans are suboptimal. Simple subtraction of emission data from the transmission sinogram and FBP reconstruction of emission image ignore the Poisson nature of the measured data. Although these methods give satisfactory results for brain scans [2], they perform much worse in the thorax where attenuation is more nonuniform and its correction is more important. This paper presents Maximum Penalized Likelihood (MPL) methods to improve the quality of the image reconstructions in simultaneous transmission/emission scans.

We compare conventional and statistical methods by use of bias versus standard deviation plots. We also present reconstructed images for comparison. The effects of resolution mismatch between attenuation and emission images are also determined.

\section{The MOdeL}

Let $\lambda=\left[\lambda_{1}, \ldots, \lambda_{p}\right]$ denote the vector of unknown emission counts originating from image pixels, and $\mu=$ $\left[\mu_{1}, \ldots, \mu_{p}\right]$ be the vector of linear attenuation coefficients (having units of inverse length). Let $y^{T}=\left[y_{1}^{T}, \ldots, y_{N}^{T}\right]$ and $y^{E}=\left[y_{1}^{E}, \ldots, y_{N}^{E}\right]$ denote the vector of measured counts in transmission and emission window sinograms respectively. We assume that the $y_{i}^{T}$ and $y_{i}^{E}$ are realizations of statistically independent random variables having Poisson distributions and with expectations $\bar{y}_{i}^{T}$ and $\bar{y}_{i}^{E}$ :

$$
\begin{aligned}
& \bar{y}_{i}^{T}(\lambda, \mu)=b_{i} e^{-l_{i}(\mu)}+k_{T} p_{i}(\lambda) e^{-l_{i}(\mu)}+r_{i}^{T} \\
& \bar{y}_{i}^{E}(\lambda, \mu)=p_{i}(\lambda) e^{-l_{i}(\mu)}+r_{i}^{E}
\end{aligned}
$$

for $i=1 \ldots N$, and where

$$
p_{i}(\lambda)=\sum_{j=1}^{p} a_{i j} \lambda_{j} \text { and } l_{i}(\mu)=\sum_{j=1}^{p} g_{i j} \mu_{j} .
$$

The $a_{i j}$ contain factors representing the tomographic system geometry, scan time, detector efficiencies, and dead time correction factors. The $g_{i j}$ have units of length and represent attenuation geometry. Here $b_{i}$ are the blank scan counts, $p_{i}$ and $l_{i}$ are the projections of true emission and attenuation parameters, $r_{i}^{T}$ and $r_{i}^{E}$ are background events in their respective windows, $k_{T}$ is the fraction of emission counts in the transmission window.

Our final goal is to estimate $\lambda$ from the two set of measurements. But, an estimate for the attenuation map $\mu$ is also found in the process for statistical reconstruction methods.

0-7803-3534-1/97 10.00@1997IEEE 


\section{iII. Resolution Matching}

Generally, for reducing noise in the transmission scan, sinogram smoothing is employed. Even though this reduces the noise in the attenuation correction factors, it introduces a resolution mismatch between two scans. It is well known that when the transmission data is smoother than the emission data, the outcome is serious artifacts at the edges which can be misinterpreted as pathological changes [3], [4]. Particularly, the photon activity at or around the edges of the attenuation map will be underestimated at the high attenuation side, and overestimated at the low side, an artifact caused by oversmoothed attenuation map.

We found that using smooth ACFs in statistical methods causes a similar effect on the emission image, as might be expected. Since, we include a biased (blurred) estimate of survival probabilities $e^{-l_{i}}$ in the emission data model, this bias propagates to the final image, causing visually disturbing images. This is illustrated in Figure 1.

It is possible to overcome the mismatch problem in non-statistical reconstructions by smoothing the emission sinogram to the resolution of the attenuation correction factors [3]. However, when one uses statistical methods, the same procedure cannot be done, since it will destroy the Poisson nature of the data. Consequently, for the statistical methods, it is necessary to use attenuation reconstructions having as small bias as possible. This suggests use of nonquadratic penalties which result in sharper edges in the attenuation map [5]. The performance of statistical methods may degrade faster than FBP with use of smoother ACFs.

\section{Methods of Reconstruction}

\section{A. Attenuation Map}

To precisely estimate the attenuation map, we need to get an initial estimate of emission contamination in the transmission window. Conventional methods estimate this contamination from either a preceding emission scan or the emission window measurements in the simultaneous scan. Then, the emission contamination estimate is subtracted from the transmission measurements, or included in the statistical model depending on the method to be used for ACF estimation.

We used three different methods for ACF computation, described as follows.

\section{A.1 RAW}

For this type, we ignore the emission contamination in the transmission window, and divide transmission measurements to blank scan data to yield the survival probabilities along lines of response. We, then smooth with a 2-D gaussian kernel in the sinogram domain to achieve a target resolution:

$$
\widehat{e^{-l_{i}}}=\operatorname{smooth}\left\{\left(y_{i}^{T}-r_{i}^{T}\right) / b_{i}\right\} .
$$

Note that we do not need to obtain the image domain attenuation map for this method. If we need to refer to $\hat{\mu}$ for this method, we find it by:

$$
\hat{\mu}=\mathrm{fbp}\left\{-\log \left(\widehat{e^{-l_{i}}}\right)\right\},
$$

where the FBP is performed by a ramp filter with cutoff at Nyquist frequency. The back projection is done pixeldriven with linear interpolation in the radial direction of the sinogram domain, so that no additional blurring is introduced.

\section{A.2 SUBTRACTION (SUB)}

We subtract the emission contamination estimated by $k_{T}\left(y^{E}-r^{E}\right)$ and randoms estimate from transmission data and divide by blank scan. Then, the resulting sinogram is 2-D smoothed to achieve various resolutions. In other words:

$$
\widehat{e^{-l_{i}}}=\operatorname{smooth}\left\{\left(y_{i}^{T}-r_{i}^{T}-k_{T}\left(y_{i}^{E}-r_{i}^{E}\right)\right) / b_{i}\right\}
$$

Similarly, the attenuation map estimate is found using FBP with a ramp filter.

\section{A.3 MPL}

To use a statistical penalized likelihood method to reconstruct the attenuation map, we estimate the emission contamination in the transmission window from $y^{E}$ and include it in the statistical model for $y^{T}$ as part of the background events. Then, we estimate $\mu$ using the penalized likelihood objective function for the transmission problem:

$$
\begin{gathered}
\hat{\mu}=\arg \max _{\mu} \Phi\left(\mu ; y^{T}\right), \\
\Phi\left(\mu ; y^{T}\right)=L\left(\mu ; y^{T}\right)-\beta R(\mu),
\end{gathered}
$$

where $L\left(\mu ; y^{T}\right)$ is the log-likelihood function given by:

$$
L\left(\mu ; y^{T}\right)=\sum_{i=1}^{N} y_{i}^{T} \log \left(b_{i} e^{-l_{i}(\mu)}+\hat{r}_{i}^{T}\right)-\left(b_{i} e^{-l_{i}(\mu)}+\hat{r}_{i}^{T}\right),
$$

where $\hat{r}_{i}^{T}=r_{i}^{T}+k_{T}\left(y_{i}^{E}-r_{i}^{E}\right)$ and the objective includes a roughness penalty function, given in general by:

$$
R(\mu)=\sum_{j} \frac{1}{2} \sum_{k} w_{j k} \psi\left(\mu_{k}-\mu_{j}\right) .
$$

Ordinarily $w_{j k}=1$ for horizontal and vertical neighboring pixels, $w_{j k}=1 / \sqrt{2}$ for diagonal neighboring pixels, and $w_{j k}=0$ otherwise. The parameter $\beta$ controls the level of smoothing. In our reconstructions, we adopt the modification described in [6] to achieve more uniform spatial resolution.

The potential function $\psi$ is a measure of similarity between pixels in the reconstructed image. For the quadratic penalty (MPL-Q), we use:

$$
\psi(x)=x^{2} / 2,
$$

and for non-quadratic penalty reconstructions (MPL-N), we focus on one of the penalties proposed in [5]:

$$
\psi(x)=\delta^{2}[|x / \delta|-\log (1+|x / \delta|)]
$$


The latter penalty approaches the quadratic penalty as $\delta \rightarrow \infty$, whereas for finite values of $\delta$, different degrees of edge preservation can be achieved.

The MPL methods can also be called "reprojected" ACF computation since the line integrals are found by projecting the attenuation maps, or $\hat{l}_{i}(\hat{\mu})=\sum_{j=1}^{p} g_{i j} \hat{\mu}_{j}$. These projections are then used to find the ACFs.

\section{B. Emission Image}

To reconstruct the emission image, there are again (at least) two alternatives: FBP and MPL reconstructions.

\section{B.1 FBP}

We smooth the randoms-corrected emission measurements $y^{E}-r^{E}$ with a gaussian 2-D kernel to achieve the same resolution as the $\hat{\alpha}_{i} \stackrel{\text { def }}{=} \widehat{e^{-l_{i}}}$ in the sinogram domain. The kernel size is determined from the best gaussian match to the survival probabilities $\hat{\alpha}$. After attenuation is corrected by division, the emission image is reconstructed by FBP with a ramp filter. In other words:

$$
\begin{gathered}
\hat{p}_{i}=\frac{\operatorname{smooth}\left(y_{i}^{E}-r_{i}^{E}\right)}{\hat{\alpha}_{i}}, \\
\hat{\lambda}=\operatorname{fbp}\{\hat{p}\} .
\end{gathered}
$$

\section{B.2 MPL}

We include the survival probability estimates $\hat{\alpha}_{i}$ in the statistical model for emission data as part of the calibration factors. Then, the penalized likelihood problem can be formulated as:

$$
\hat{\lambda}=\arg \max _{\lambda} L\left(\lambda ; y^{E}, \hat{\alpha}\right)-\beta R(\lambda),
$$

where the log-likehood function is,

$$
\left.L\left(\lambda ; y^{E}, \hat{\alpha}\right)=\sum_{i=1}^{N} y_{i}^{E} \log \left(p_{i}(\lambda) \hat{\alpha}_{i}+r_{i}^{E}\right)-\left(p_{i}(\lambda) \hat{\alpha}_{i}+r_{i}^{E}\right)\right),
$$

and $R(\lambda)$ is the quadratic penalty function (MPL-Q).

We again use a modification of the weights $w_{j k}$ to achieve near-uniform resolution [6]. The parameter $\beta$ is varied to obtain different levels of smoothing.

\section{Performance Simulations}

\section{A. The Phantom and The Parameter Values}

To compare different methods, we used a synthetic attenuation map and emission distribution shown in top left corners of Figures 5 and 6 as $\mu^{\text {true }}$ and $\lambda^{\text {true. The }}$ attenuation map represents a human thorax cross section with linear attenuation coefficients $0.16 \mathrm{~cm}^{-1}, 0.096$ $\mathrm{cm}^{-1}, 0.025 \mathrm{~cm}^{-1}$, for bone, soft tissue and lungs, respectively. The emission image represents sample activity in the same cross section with values 1,2 and 4 for lungs, soft tissue and heart, respectively. The pixel size is 4.22 $\mathrm{mm}$. We simulated PET transmission and emission scans with 160 radial bins and 192 angles uniformly spaced over $180^{\circ}$. The $a_{i j}$ factors corresponded to $6 \mathrm{~mm}$ wide strip integrals with $3 \mathrm{~mm}$ center to center spacing, which is an approximation to ideal line integral that accounts for finite detector width.

We set the number of counts of transmission scan to 2 million and of emission scan to 1 million. The randoms rate were $10 \%$ in both scans. The randoms rate in emission window is usually higher than $10 \%$, but it is possible to reduce it by using weaker rod sources [1]. Emission scan can also be done separately after removing the rod sources in which case the $\mathrm{T}+\mathrm{E}$ scan is termed postinjection transmission scan. This does not change our formulation if we assume no patient motion in between these two scans and consider the radioactive decay. Detector efficiencies and blank scan sinograms were assumed to be uniform. Deadtime was ignored in the simulations. There was emission contamination of $10 \%\left(k_{T}\right)$ in the transmission window.

We generated $M=100$ realizations of pseudorandom Poisson transmission and emission measurements according to (1), then reconstructed using previously mentioned methods. For the MPL reconstructions, we used the grouped ascent algorithm for transmission [7], and SAGE algorithm for emission reconstructions [8].

\section{B. Results}

We present the average bias versus standard deviation estimate graphs for both attenuation and emission image reconstructions in Figures 2, 3 and 4. In these figures, the horizontal axis values are obtained from the sample mean image of $M$ reconstructions. We take the average of absolute differences between the true image and the sample mean within a region of interest $(W)$. The values are normalized by the average value of the true image in that region. Let $\theta^{n}, n=1 . . M$ denote the reconstructions obtained from $M$ realizations, and let $\bar{\theta}$ denote their sample mean, then:

$$
b(\theta)=\frac{\sum_{j \in W}\left|\bar{\theta}_{j}-\theta_{j}^{\text {true }}\right|}{\sum_{j \in W}\left|\theta_{j}^{\text {true }}\right|} \times 100 \%,
$$

is the estimate of the average bias. We choose the window $W$ to be a central rectangular region containing both lungs for bias estimates.

The vertical axis values are the average standard deviation estimate found from $M$ realizations in another region of interest, i.e:

$$
\sigma(\theta)=\frac{1}{|W|} \sum_{j \in W} \sqrt{\frac{1}{M-1} \sum_{n=1}^{M}\left(\theta_{j}^{n}-\bar{\theta}_{j}\right)^{2}} .
$$

The window $W$ for the attenuation map is the same as the bias window, whereas for emission standard deviation estimates, we choose a smaller region around the cardiac activity area.

The plot in Figure 2 clearly indicates that both MPL methods have better performance than the subtraction method for transmission processing. The raw estimate is severely biased as expected. Also, MPL-N has a better bias-variance trade-off than MPL-Q reconstruction. Nonquadratic penalties appear to be preferable for transmission reconstruction. 
In Figure 4, we used an emission MPL-Q reconstruction with fixed $\beta=2^{6.4}$ giving a resolution of about 12 $\mathrm{mm}$. Thus, the difference in the bias values are only due to different transmission reconstructions. The bias increases as we use smoother attenuation maps, but the standard deviation estimates do not go down as much. It can be seen that MPL-N transmission followed by MPL$\mathrm{Q}$ emission reconstruction seems to give the best result.

Figure 3 presents a similar plot for FBP emission reconstructions. In this case, the resolutions of emission data are matched to attenuation resolution and no further smoothing is done. Thus, actually initial points on the graph are very noisy which results in the strange curves in the plot. This is due to the fact that, even the mean images corresponding to these reconstructions are noisy, which show up in the bias estimate. Actually, in the ideal case, we should put error bars to show the accuracy of our bias and standard deviation estimates. However, since the resolutions are matched, there should not be any contribution from systematic artifacts at the edges unlike MPL-Q estimates. The bias values in this case are proportional to FWHM values for reasonably smooth reconstructions. The plot indicates that FBP method is inferior to MPL-Q for low values of bias. But, for higher values of bias $(\geq 13 \%)$, FBP seems comparable to MPL-Q because of the resolution mismatch problem in the statistical method.

We also present sample reconstruction images from a single realization. In Figure 5, the attenuation maps can be observed. The MPL-N looks much better than MPL-Q or SUB reconstructions. Here, note that the resolutions of the last two estimates are almost matched, but the first one is sharper at the edges. Even then, MPL-N looks less noisy.

Emission images are presented in Figures 6 and 7 . Overall, MPL-Q estimates look better qualitatively than FBP ones which contain disturbing streak artifacts. The reconstructions with RAW ACFs have systematic negative bias. Visually, the best one is the combination of MPL-N for attenuation and MPL-Q for emission, which seems to reduce the noise inside the lungs appearing as hot spots in other reconstructions. This noise is apparently coming from noisy transmission data, which is successfully reduced in the MPL-N attenuation map estimate.

\section{Conclusion}

We investigated the use of statistical image reconstruction methods for simultaneous transmission/emission PET imaging. We compared the conventional methods with statistical ones, as well as hybrid ones. The MPL reconstructions are shown to outperform conventional methods for reasonable values of bias. Non-quadratic penalties yield better results than quadratic ones since they allow for sharp edges and yet still reduce the noise. We have used MPL-N only for transmission. Its use for emission may improve the image quality further.

In this work, we only used conventional methods with linear smoothing in the sinogram domain. For future work, the statistical methods should be compared with
FBP with segmentation of the attenuation map. We still suspect that statistical MPL methods will outperform that approach, since segmentation algorithms generally misclassify important number of pixels due to noise. But, some sort of soft-segmentation might work. Meanwhile, we are in search of methods to obtain sharper but less noisy reconstructions of attenuation maps to further reduce the transmission component of the image noise.

The next step in this area is to use the first emission image to estimate the emission contamination in the transmission window and reconstruct $\mu$ and $\lambda$ in alternation until more satisfactory results are obtained. Another alternative we are investigating is to jointly estimate $\mu$ and $\lambda$ from the two set of measurements $y^{E}$ and $y^{T}$.
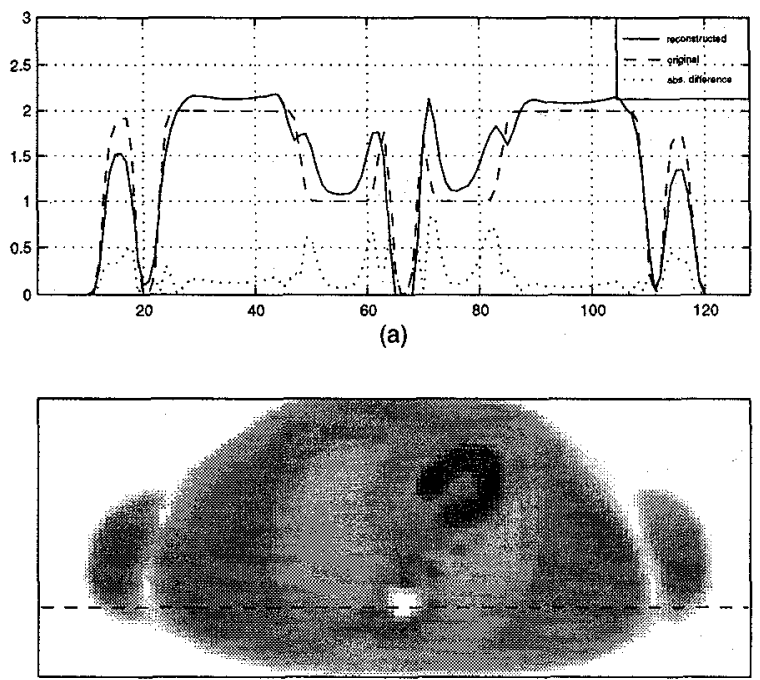

(b)

Fig. 1. The artifacts casued by mismatch of resolutions. Attenuation is reconstructed from noiseless data using quadratic penalty with $\log _{2} \beta=7.6$ resulting in a psf of FWHM $14 \mathrm{~mm}$. The emmission image is reconstructed again from noiseless data using quadratic penalty with $\log _{2} \beta=3.0$ yielding a psf of FWHM $6 \mathrm{~mm}$.

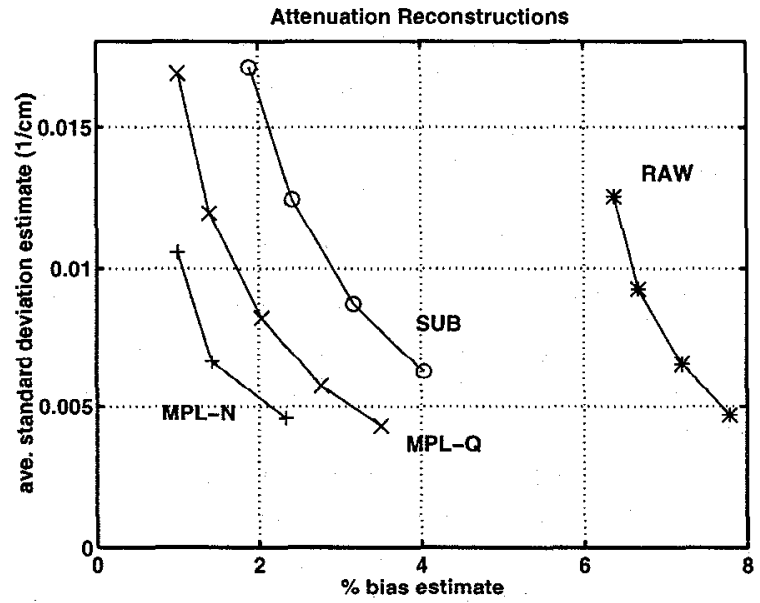

Fig. 2. Bias vs standard deviation trade-offs for attenuation map reconstructions. Labels indicate different reconstruction methods.

\section{REFERENCES}

[1] S. Meikle, D. L. Bailey, B, F. Hutton, and W. F. Jones, "Op- 


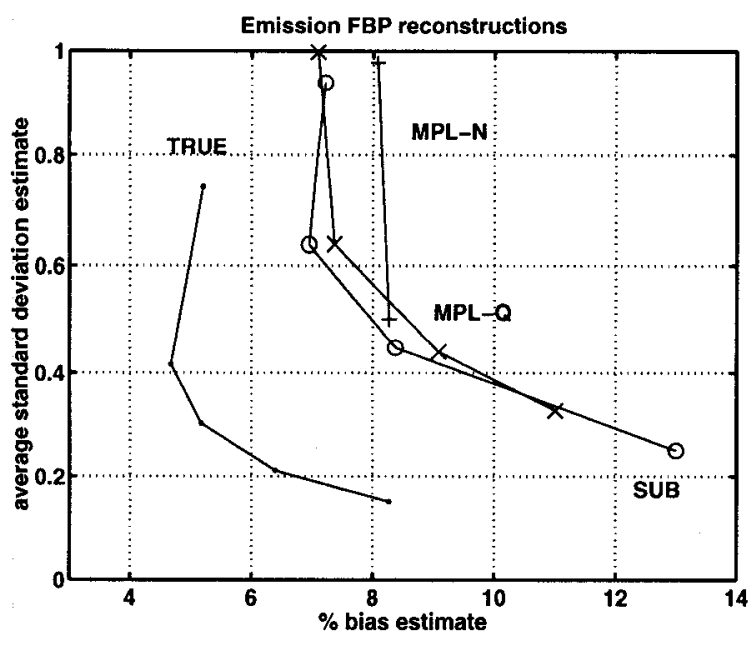

Fig. 3. Bias vs standard deviation trade-offs for FBP emission reconstructions. Labels indicate the attenuation map that is used.

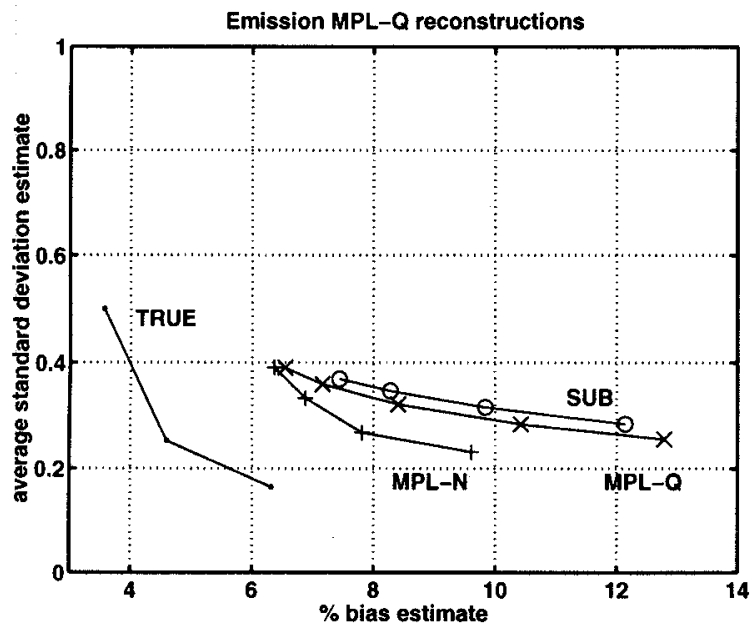

Fig. 4. Bias vs standard deviation trade-offs for quadratic penalty emission reconstructions. Labels indicate the attenuation map that is used.

timization of simultaneous emission and transmission measurements in PET," in Proc. IEEE Nuc. Sci. Symp. Med. Im. Conf., volume 3, pp. 1642-1646, 1993.

[2] M. E. Daube-Witherspoon, R. E. Carson, and M. V. Green, "Post-injection transmission attenuation measurements for PET," IEEE Tr. Nuc. Sci., vol. 35, no. 1, pp. 757-761, February 1988.

[3] A. Chatziioannou and M. Dahlbom, "Detailed investigation of transmission and emission data smoothing protocols and their
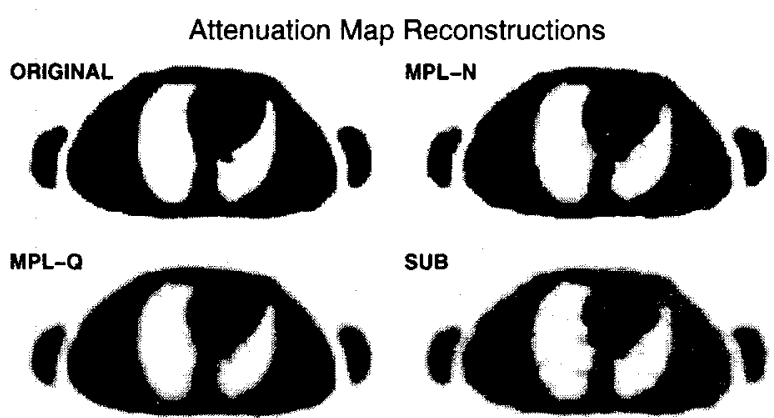

Fig. 5. Attenuation Maps obtained through various reconstructions from a single noisy measurement realization.
Emission FBP Reconstructions
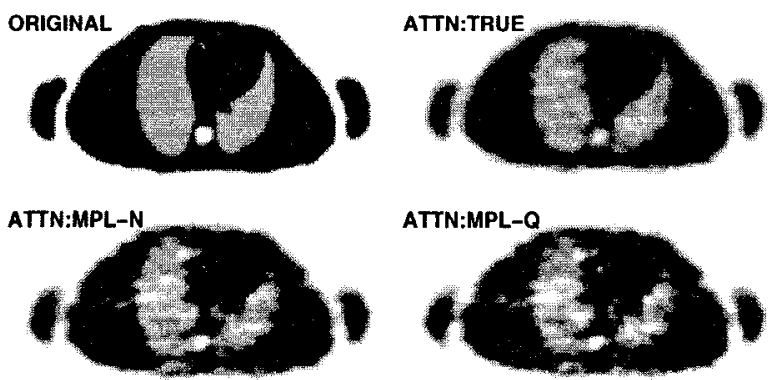

ATTN:MPL-Q
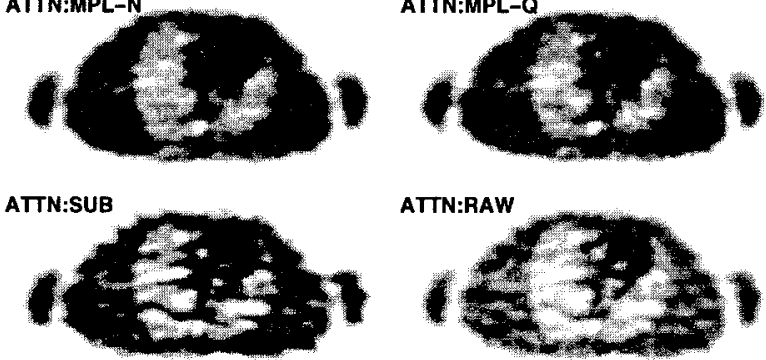

Fig. 6. FBP reconstructions obtained from a single realization.
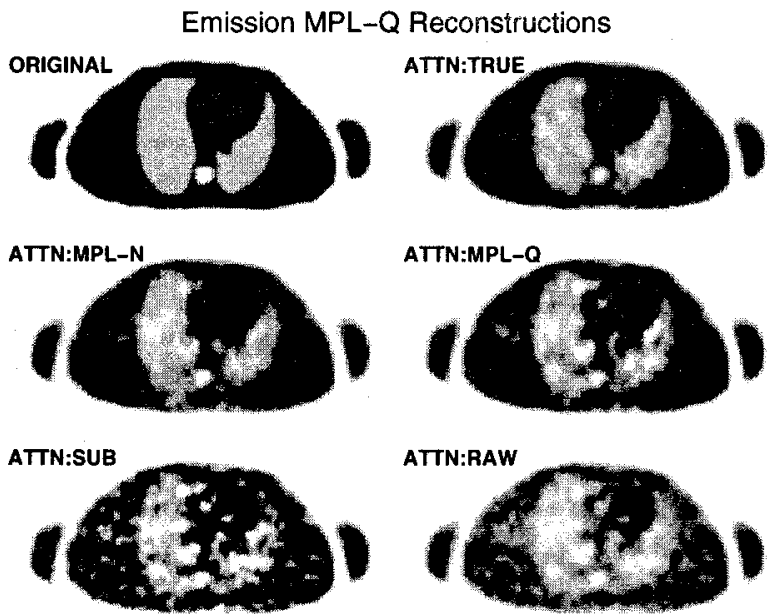

Fig. 7. MPL-Q reconstructions obtained from the same noisy data as the FBP reconstructions.

effects on emission images," IEEE Tr. Nuc. Sci., vol. 43, no. 1, pp. 290-4, February 1996.

[4] S. R. Meikle, M. Dahlbom, and S. R. Cherry, "Attenuation correction using count-limited transmission data in positron emission tomography," $J . N u c$. Med., vol. 34, no. 1, pp. 143-150, January 1993.

[5] K. Lange, "Convergence of EM image reconstruction algorithms with Gibbs smoothing," IEEE Tr. Med. Im., vol. 9, no. 4, pp. 439-446, December 1990. Corrections, June 1991.

[6] J. A. Fessler and W. L. Rogers, "Uniform quadratic penalties cause nonuniform image resolution (and sometimes vice versa)," in Proc. IEEE Nuc. Sci. Symp. Med. Im. Conf., volume 4, pp. 1915-1919, 1994

[7] J. A. Fessler, E. P. Ficaro, N. H. Clinthorne, and K. Lange, "Grouped-coordinate ascent algorithms for penalized-likelihood transmission image reconstruction," IEEE Tr. Med. Im., 1996. To appear.

[8] J. A. Fessler and A. O. Hero, "Penalized maximum-likelihood image reconstruction using space-alternating generalized EM algorithms," IEEE Tr. Im. Proc., vol. 4, no. 10, pp. 1417-29, October 1995. 